\title{
Les surcotes et les décotes marines à Brest, étude statistique et évolution
}

\author{
Romuald BOULIGAND, Paolo Antonio PIRAzzoli \\ Institut de géographie, université de Paris-1, laboratoire de géographie physique, Ura 141-CNRS, \\ place Aristide-Briand, 92190 Meudon-Bellevue, France
}

(Reçu le 23 janvier 1998, révisé le 17 juin 1998, accepté le 20 juin 1998)

\begin{abstract}
Positive and negative sea surges at Brest. Sea surges (positive or negative) are short-period events (several hours to several days) among the most extreme oceanic phenomena resulting from climatic variability. A statistical study of hourly tide-gauge records at Brest does not allow any clear trend in long-term variations of these extreme sea levels to be detected. However, the frequency of extreme positive sea surges has increased recently (1953-1994), whereas extreme and sub-extreme negative sea surges has decreased. Such trends for the highest values strengthen the prevalence of positive sea surges over negative ones. The general evolution appears to be organised around several time scales, with a strong interannual variability superimposed on periods of amplification or regression of decennial order. (C) Elsevier, Paris I Ifremer / Cnrs / Ird
\end{abstract}

sea level / positive and negative sea surges / frequency / trend / Brest

Résumé - Les surcotes et les décotes sont des événements de courtes périodes (quelques heures à quelques jours) parmi les manifestations océaniques les plus fortes que l'on puisse attribuer à la variabilité climatique. L'étude menée sur la longue série marégraphique de Brest ne permet pas de discerner une tendance claire dans les variations à long terme de ces phénomènes. Néanmoins, la fréquence des surcotes extrêmes a augmenté lors de la période récente (1953-1994), alors que les décotes extrêmes et subextrêmes ont suivi une évolution inverse. Cela a pour effet de renforcer la prédominance, déjà forte, des surcotes sur les décotes pour les valeurs les plus élevées. Il apparaît aussi que leur évolution s'organise selon plusieurs échelles de temps, puisqu'à une variabilité interannuelle très forte se superposent, à l'échelle décennale, des périodes d'amplification et de régression de ces niveaux. ( ) Elsevier, Paris / Ifremer / Cnrs / Ird

niveau marin / surcotes et décotes marines / fréquence / évolution / Brest

\section{INTRODUCTION}

Le réchauffement global du climat qui semble s'opérer actuellement se manifesterait plutôt par un accroissement des effets de la variabilité climatique que par une élévation du niveau de la mer $[4,10,13]$. Définies comme la différence entre la marée observée et la marée astronomique prévue au même instant, les surcotes et les décotes marines sont en partie contrôlées par cette variabilité, puisqu'elles témoignent de l'influence des facteurs météorologiques, essentiellement la pression atmosphérique et le vent, sur le niveau momentané du plan d'eau.
Les contraintes exercées par la pression atmosphérique entraînent une réponse «statique » de la mer qui réagit comme un baromètre inversé. Une élévation de $1 \mathrm{~cm}$ correspond à une baisse de pression d'environ $1 \mathrm{hPa}$. Cette corrélation, bien établie en plein océan, est cependant plus difficilement appréciable le long des côtes à cause des effets hydrodynamiques, qui dependent de la configuration et de la topographie côtières et peuvent renforcer ou atténucr cette réponsc «statique ». Concrètement, lorsque la pression est inférieure à $1013 \mathrm{hPa}$ (valeur moyenne au niveau de la mer), la surface de la mer se soulève et forme une intumescence, alors qu'en présence 
de hautes pressions, on observe une concavité. On parle alors de surcote ou de décote barométrique.

En produisant des forces de frottement sur la surface de la mer et en transmettant de l'énergie mécanique aux masses d'eau, le vent induit des variations du niveau marin qui peuvent provoquer également des surcotes ou des décotes. Cette action du vent, qui se combine aux effets de la pression, dérive de trois paramètres : la direction d'où vient le vent (qui peul provoquer un afflux d'eau vers la côte, générant ou amplifiant une surcote, ou bien produire un reflux d'eau et donc donner une décote), sa vitesse (qui amplifie le plus souvent ces effets) et sa durée (qui contrôle la durée du phénomène).

Plusieurs auteurs ont essayé d'estimer la fréquence des niveaux extrêmes de la marée. Alors que le phénomène des surcotes et décotes est prédominant sur les littoraux des mers à faible marnage $[7,8]$, sur les côtes à marée importante, la part des surcotes ou des décotes ne représente qu'une composante, souvent faible, de la marée observée. Ce n'est donc que lors de cas extrêmes intervenant lors de pleines mers ou de basses mers de fort coefficient que leurs effets deviennent remarquables. Pugh et
Vassie [9] ont montré que les surcotes météorologiques et la marée astronomique sont largement indépendants, ce qui permet de calculer séparément leurs probabilités d'après l'analyse des observations horaires de la marée. Simon [11] préfèrc limiter l'analyse à l'ćtude des écarts entre les hauteurs des pleines mers observées et prédites (afin d'éviter les défauts de calage horaires entre prévisions et observations). En combinant les lois de probabilités des hauteurs de pleines mers prédites et des valeurs des surcotes observées dans 15 ports de France, il aboutit à une cartographie des lignes d'égales hauteurs atteintes par la mer avec une période de retour donnée pour tous les points des côtes françaises de l'Atlantique et de la Manche.

L'approche de Simon, extrêmement efficace pour ce qui concerne les probabilités d'inondations littorales, n'utilise cependant qu'une partie des données disponibles et ne permet pas une étude approfondie de l'évolution de ces phénomènes. Or ces derniers risquent de subir des variations associées aux changements climatiques globaux (prévus par certains modèles) susceptibles de modifier les temps de retour des niveaux extrêmes. Il devenait

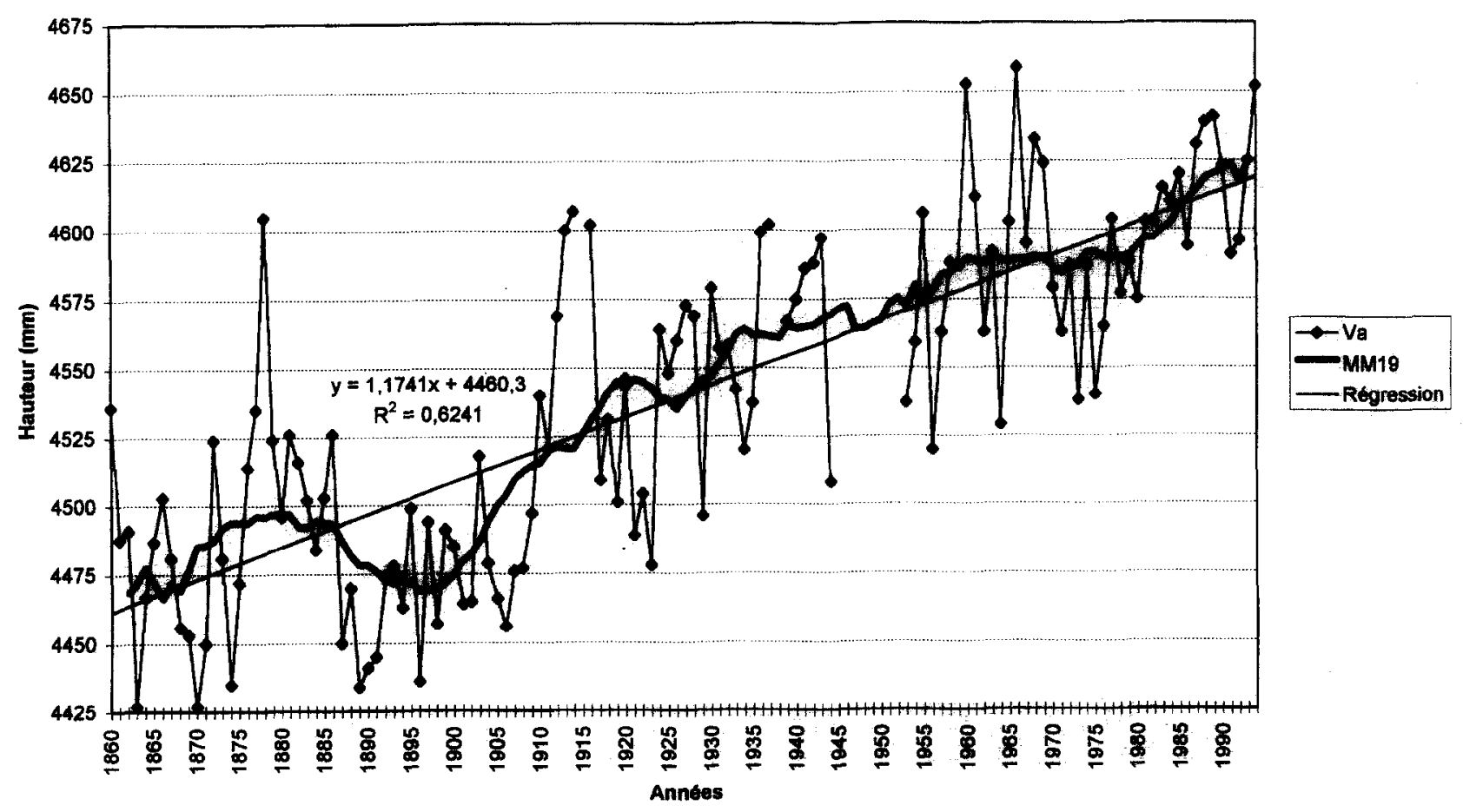

Figure 1. Niveau moyen de la mer à Brest (1860-1995) : valeurs moyennes annuelles (Va), moyenne mobile sur 19 ans (extrapolée en début et fin de série) (MM19) et droite de régression.

Figure 1. Mean sea level at Brest (1860-1995): annual means (Va), 19-year running mean (extrapolated) (MM19) and linear regression line. 
donc intéressant d'analyser, sur une longue série, la fréquence et la répartition des surcotes et des décotes. Afin d'être le plus rigoureux possible dans notre approche statistique, nous avons choisi d'étudier les principales caractéristiques des surcotes et des décotes horaires de toutes les marées disponibles pour Brest depuis 1860 (grâce au Shom) selon plusieurs échelles de temps et pour différents niveaux d'intensité.

\section{TRAITEMENT PRÉLIMINAIRE DES DONNÉES}

La série marégraphique de Brest est connue comme la plus ancienne de France et constitue une référence à l'échelle mondiale. Les premières observations sur échelle de marée remontent à 1807 . Le premier marégraphe a été installé en 1860 et un marégraphe numérique est utilisé depuis 1993. Les lacunes depuis 1860 sont limitées à environ $9 \%$ (essentiellement les années 1915, 1938 et de mai 1944 à décembre 1952). Nous avons uti- lisé pour cette étude l'ensemble des données numérisées disponibles entre mai 1860 et mars 1995.

Le calcul de la marée astronomique a été effectué à l'aide du logiciel Predit du Shom, qui fournit un fichier de valeurs horaires pour la période choisie. Avant de procéder au calcul des surcotes-décotes, il a fallu cependant déduire des observations les variations observées du niveau moyen de la mer. Ces dernières témoignent d'une élévation moyenne de $1,17 \pm 0,03 \mathrm{~mm} / \mathrm{an}$ $(1 \sigma)$, mais avec de nombreuses oscillations (figure l). Une moyenne mobile sur 19 ans (ou sur un nombre plus faible d'années en début et en fin de série et pour les données manquantes) permet de lisser ces oscillations tout en filtrant les influences du cycle de la déclinaison lunaire de 18,61 ans.

Les surcotes-décotes horaires ont été enfin calculées avec le logiciel Surcote (élaboré par M. Ba, pour l'Ura 141 du CNRS et fonctionnant avec le langage et la procédure du Statistical Analysis System - SAS). Les listes horaires des surcotes-décotes ont été ensuite dépouillées. Cette

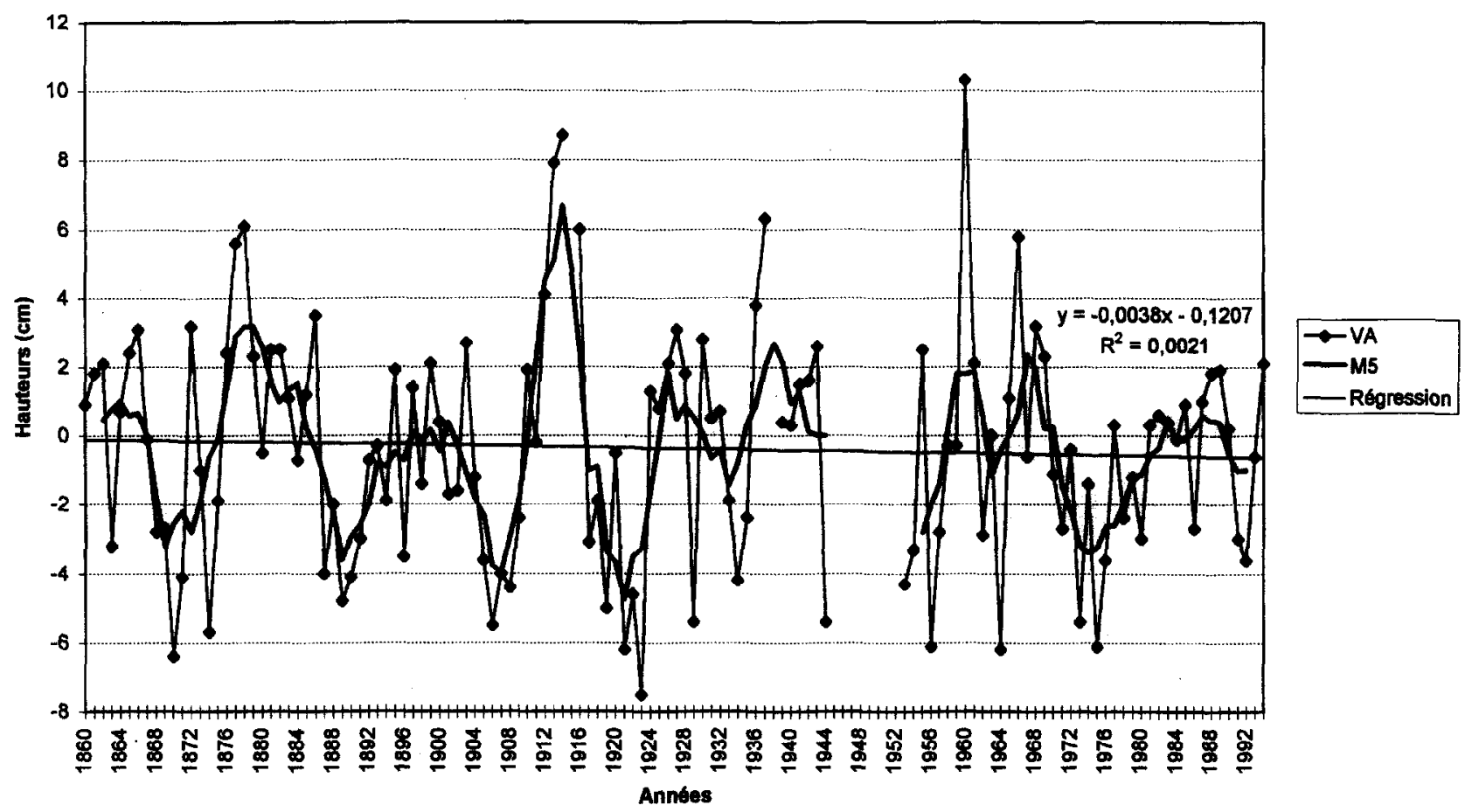

Figure 2. Surcotes-décotes horaires moyennes annuelles à Brest (1860-1995) : valeurs annuelles (Va), moyennes mobiles sur 5 ans (M5) et droite de régression.

Figure 2. Mean annual positive-negative hourly sea surges at Brest (1860-1995): annual values (Va), 5-year running mean (M5) and linear regression line. 
vérification a mis en évidence de nombreuses séries de 5 6 surcotes immédiatement suivies par 5-6 décotes, puis de nouveau 5-6 surcotes et 5-6 décotes, etc. Ces fluctuations (fréquentes, entre autres, en 1907-1909, 1912, 1961 ct 1963), qui ne correspondent visiblement pas à des seiches, semblent résulter de légers décalages entre la montre du marégraphe et le temps des prévisions, ou d'un mauvais fonctionnement de l'instrument, ou d'erreurs de numérisation. Nous les avons exclues de cette étude préliminaire. Le nombre de valeurs horaires finalement retenues pour l'analyse statistique est de 1084410 surcotesdécotes, distribuées dans 126 années utiles.

\section{3. ÉVOLUTION SÉCULAIRE DES SURCOTES- DECOTES}

L'évolution dans le temps de la moyenne de toutes les surcotes et décotes de Brest ainsi corrigées (figure 2) montre une relative stabilité à long terme (la légère baisse suggérée par la droite de régression n'est pas statistiquement significative), mais avec des fluctuations importantes. Ainsi, les surcotes sont prédominantes autour de $1876-1886,1910-1916,1960$ et 1965-1969, alors que les décotes prédominent en 1867-1871, 1887-1894, 1904-1909, 1917-1923 et 1970-1981. Une légère ten-

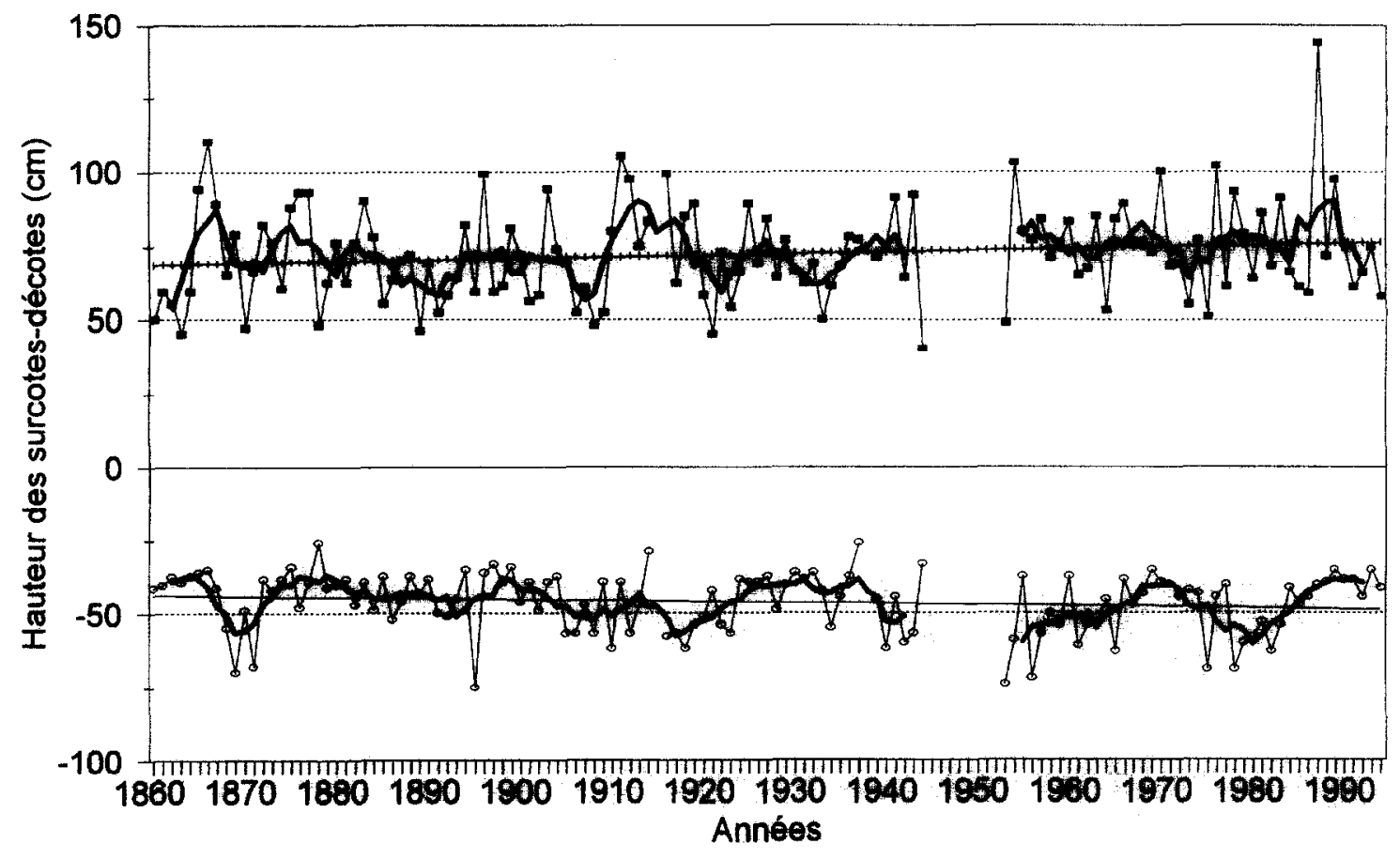

Figure 3. Surcotes et décotes horaires maximales annuelles à Brest (1860-1995) : valeurs annuelles, pentes des droites de régression avec erreurs standard et moyennes mobiles.

Figure 3. Maximum annual positive and negative hourly sea surges at Brest (1860-1995): annual means, 5-year running mean and slopes of linear regression lines with confidence interval. 
dance à l'accroissement à long terme est indiquée par les surcotes et les décotes les plus élevées (figure 3). Les surcotes maximales annuelles oscillent entre $40 \mathrm{~cm}$ (en 1944 ) et $144 \mathrm{~cm}$ (en 1987) et tendent à s'élever à la vitesse moyenne de $0,57 \pm 0,36 \mathrm{~mm} / \mathrm{an}(1 \sigma)$. Inversement, les décotes maximales oscillent entre $26 \mathrm{~cm}$ (en 1878 et en 1937) et $75 \mathrm{~cm}$ (en 1895) et tendent à s'abaisser à la vitesse de $-0,46 \pm 0,24 \mathrm{~mm} / \mathrm{an}$. Ces tendances, dont les valeurs dépassent à peine les marges d'incertitude, sont contredites par les données de la période la plus récente (1953-1994), où les décotes maximales ten- dent à s'élever de $4,1 \pm 1,3 \mathrm{~mm} / \mathrm{an}$, alors qu'aucune tendance significative n'apparaît pour les surcotes maximales annuelles. La situation est très semblable pour les $99^{\mathrm{e}}$ et $1^{\mathrm{er}}$ centiles des surcotes-décotes disponibles chaque année (figure 4). En effet, le $99^{\mathrm{e}}$ centile (qui est dépassé par $1 \%$ des valeurs de l'ensemble des surcotesdécotes, c'est-à-dire par environ $2 \%$ des surcotes de l'année considérée) tend à s'élever de $0,31 \pm 0,15 \mathrm{~mm} / \mathrm{an}$ sur toute la période considérée, mais à baisser au cours de la période la plus récente (1953-1994); inversement, le premier centile (valeur qui est dépassée par environ $2 \%$

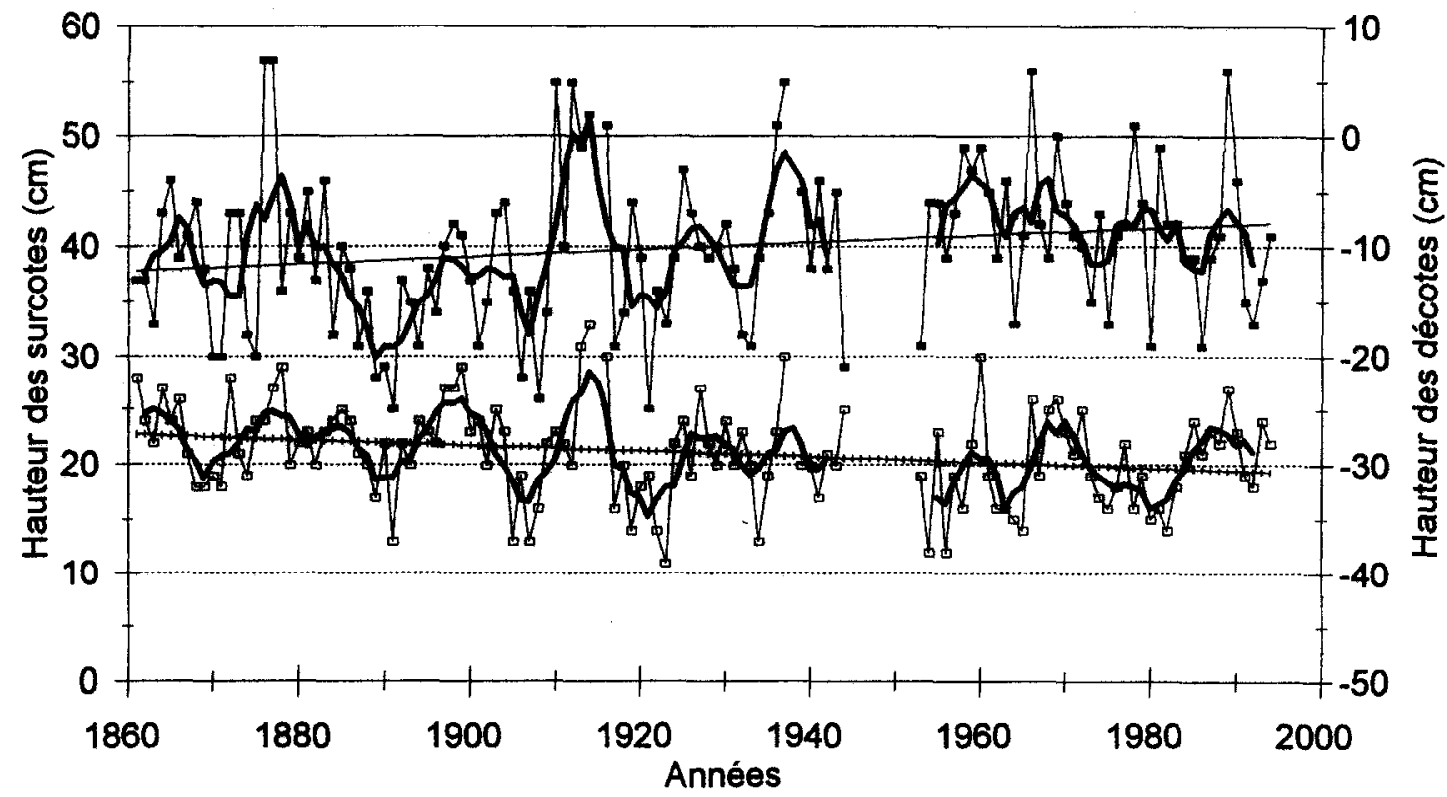

$$
\begin{array}{ll}
- \text { Surcotes (99ème centile) } & - \text { Surcotes } 99 \% \text { (moyenne } 5 \text { ans) } \\
- \text { Surcotes } 99 \%(+0,31+/-0,15 \mathrm{~mm} / \mathrm{an}) & \rightarrow \text { Décotes }(1 \mathrm{er} \text { centile) } \\
- \text { Décotes } 1 \% \text { (moyenne } 5 \text { ans) } & \text { - Décotes } 1 \%(-0,27+1-0,10 \mathrm{~mm} / \mathrm{an})
\end{array}
$$

Figure 4. Évolution des surcotes-décotes horaires extrêmes à Brest pour les $99^{\mathrm{e}}$ et $1^{\mathrm{er}}$ centiles : valeurs annuelles, moyennes mobiles et pentes des droites de régression avec erreurs standard.

Figure 4. Extreme positive and negative hourly sea surges evolution at Brest for the 99th and 1st percentiles: annual means, 5-year running mean and slopes of linear regression lines with confidence interval. 
des décotes les plus élevées de chaque année) tend à s'abaisser de $-0,27 \pm 0,10 \mathrm{~mm} / \mathrm{an}$ entre 1860 et 1994 , mais à s'élever depuis 1953.

En conclusion, aucune tendance claire de variation à long terme n'est discernable à Brest, ni pour les valeurs moyennes, ni pour les valeurs extrêmes ou subextrêmes.

\section{FRÉQUENCE DES SURCOTES-DÉCOTES}

Sur les 1084410 valeurs horaires analysées, 31735 seulement sont égales à zéro (la marée observée coïncidant avec la marée astronomique, au centimètre près) et 25564 sont manquantes. Le reste se partage entre
463779 valeurs positives (surcotes) et 563332 valeurs négatives (décotes) (figure 5). Pour des écarts de hauteur inférieurs à $\pm 20 \mathrm{~cm}$, ce sont les décotes qui sont les plus nombreuses, mais pour les valeurs les plus élevées (en valeur absolue), les surcotes prédominent nettement et sont deux fois plus élevées que les décotes. Ainsi, pour une seule décote atteignant $75 \mathrm{~cm}$, on trouve 319 cas horaires avec des surcotes atteignant ou dépassant $75 \mathrm{~cm}$.

Pour la période de retour des surcotes et des décotes extrêmes, nous avons appliqué la méthode de Gumbel [6] aux valeurs maximales annuelles. Cette méthode, fondée sur la théorie des extrêmes, permet d'obtenir les lois existant entre les niveaux maximaux et minimaux $x(\mathrm{en} \mathrm{cm})$, la probabilité $\mathrm{F}$ de chaque événement et sa période de

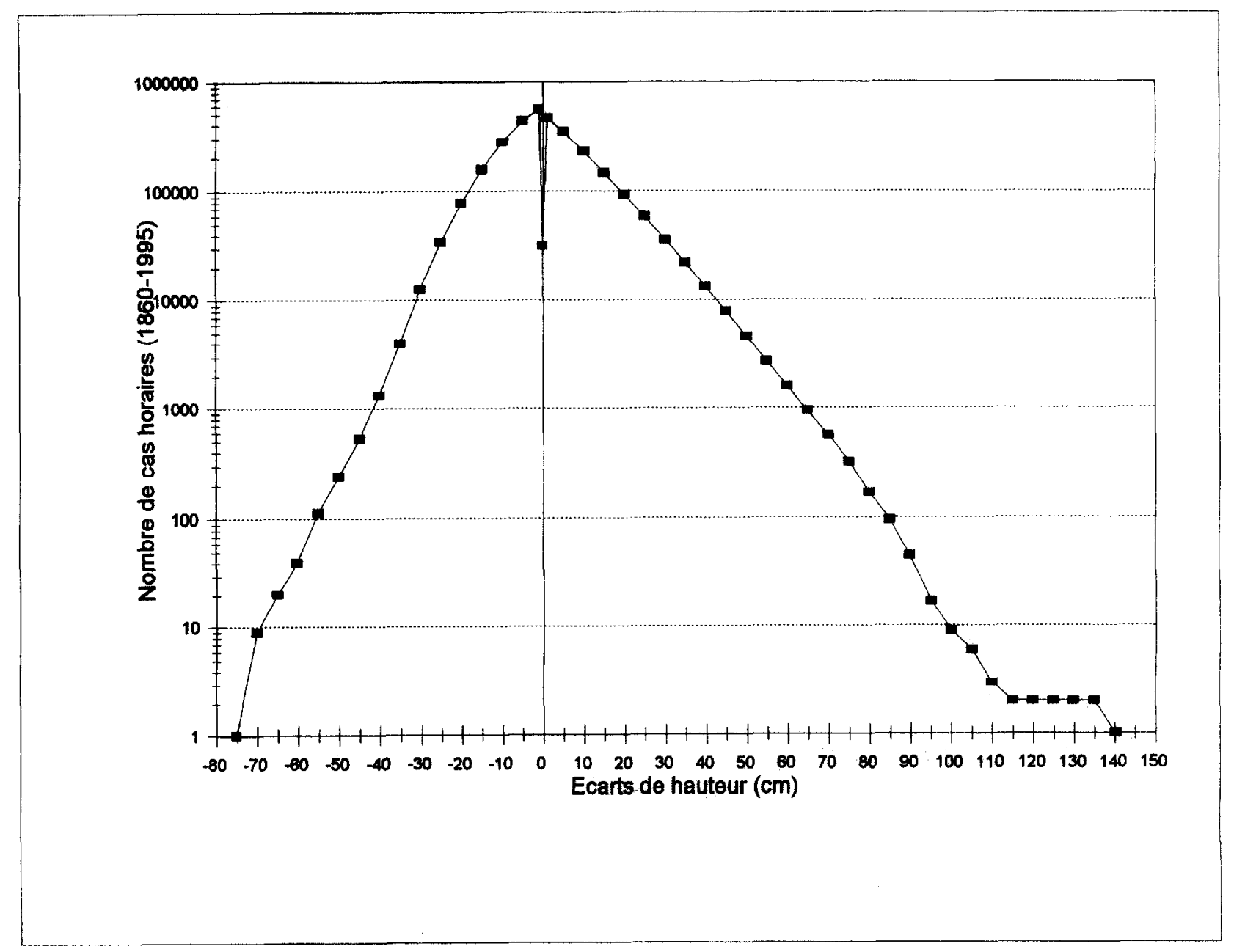

Figure 5. Répartition semi-logarithmique des surcotes et décotes horaires à Brest, qui ont atteint ou dépassé un écart de hauteur donné.

Figure 5. Semi-logarithmic distribution of positive and negative hourly sea surges at Brest, which reached or topped a certain height. 
retour $\mathbf{T}$ (en années) appliquées aux trois périodes suivantes : 1860-1944, 1953-1994 et pour l'ensemble des données disponibles entre 1860 et 1994.

La fréquence des surcotes extrêmes s'est accrue depuis 1953 (figure 6), surtout du fait de la présence, dans la dernière série, de l'événement exceptionnel de 1987. Ainsi, la période de retour d'une surcote de $144 \mathrm{~cm}$, qui aurait été de 472 ans d'après les équations (1), (2) et (3) (figure 6) entre 1860 et 1944 , serait descendue à 355 ans en considérant l'ensemble des deux périodes 1860-1944 et 1953-1994, et à seulement 158 ans en considérant la seule période 1953-1994.

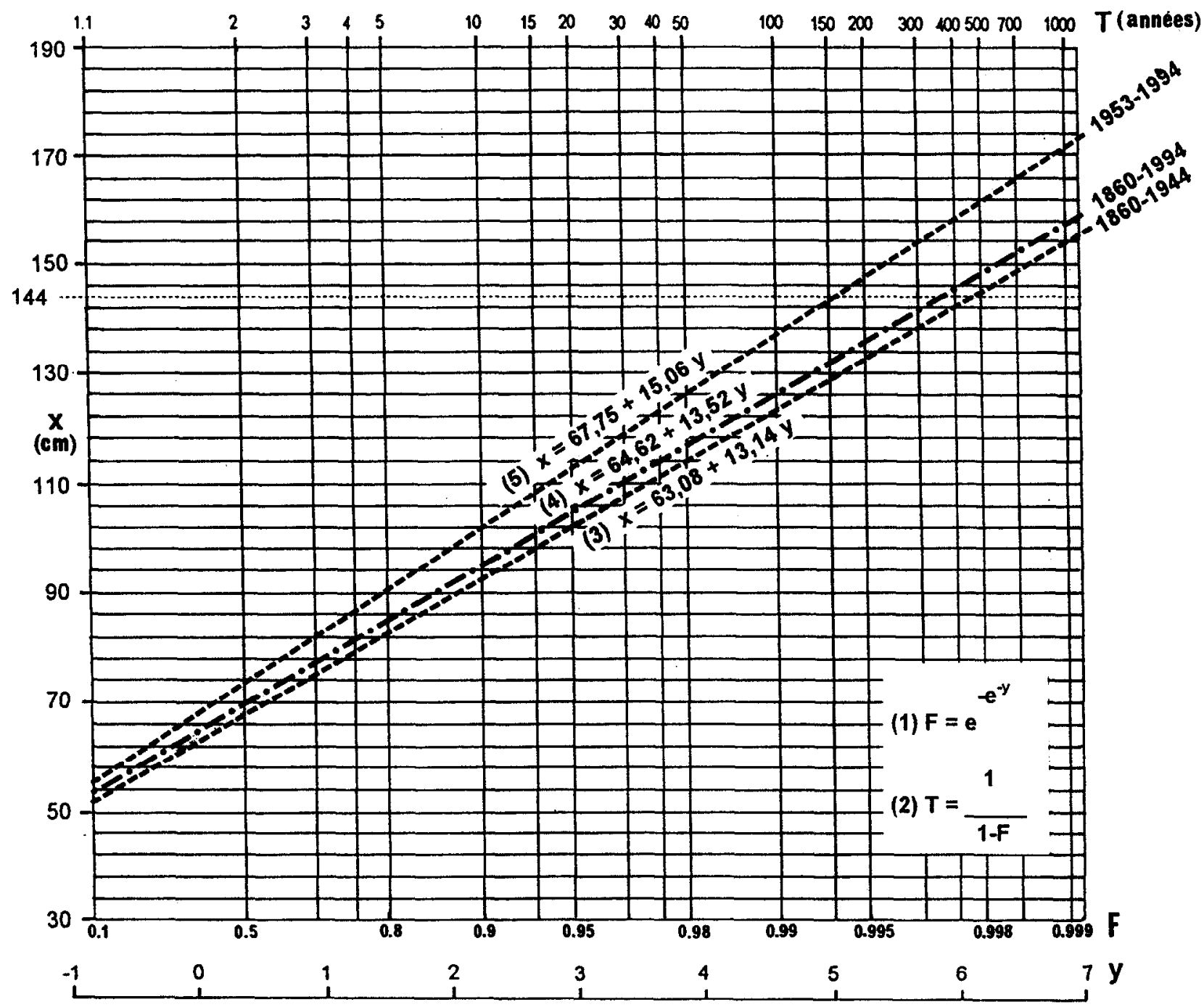

Figure 6. Courbes de fréquence (3), (4), (5) et périodes de retour (T) des surcotes horaires extrêmes annuelles (x) pour différentes périodes à Brest, d'après la méthode de Gumbel (1954). Les échelles $x, y, F(x)$ et $T(x)$ ont été tracées sur un diagramme de probabilité, dans lequel les surcotes horaires extrêmes $x$ figurent sur les ordonnées et la probabilité $F$ sur les abscisses; $y$ est une variable réduite, fonction linéaire de $x$.

Figure 6. Frequency graphs (3), (4), (5) and return periods (T) of extreme annual positive hourly sea surges (x) for different periods at Brest according to the Gumbel method (1954). The scales $x, y, F(x)$ and $T(x)$ have been plotted on a probability diagram, where the maximum annual positive hourly sea surge heights $x$ are traced along the ordinate and the probability $F$ along the abscissa; $y$ is a reduced variate, linear function of $\mathrm{x}$. 
Le caractère exceptionnel de la surcote de $144 \mathrm{~cm}$ en 1987 est clairement visible sur la figure 7 , où tous les événements avec une période de retour d'au moins 3 ans (indiqués par des points) ont été situés par rapport aux limites du domaine de probabilité $2 / 3$ (bande continuc enveloppant la droite d'équation (4)). L'écart de $34 \mathrm{~cm}$ entre le premier extrême $(144 \mathrm{~cm})$ et le second extrême $(110 \mathrm{~cm})$ est nettement supérieur à l'écart existant entre deux autres extrêmes successifs (quelques centimètres au maximum). En outre, la surcote de $144 \mathrm{~cm}$ se situe à la limite supérieure du domaine de probabilité $2 / 3$, alors que les trois extrêmes maximaux suivants $(110,105$ et $103 \mathrm{~cm}$ ) se situent légèrement en dehors de la limite inférieure du domaine de probabilité $2 / 3$.

Le côté exceptionnel de la surcote de 1987 a été peu remarqué à cause de son incidence limitée (en dehors de l'action des vagues et de la houle) sur les zones côtières basses. Ce fait est dû essentiellement au cycle de la marée qui se trouvait en phase de mortes eaux (coefficient

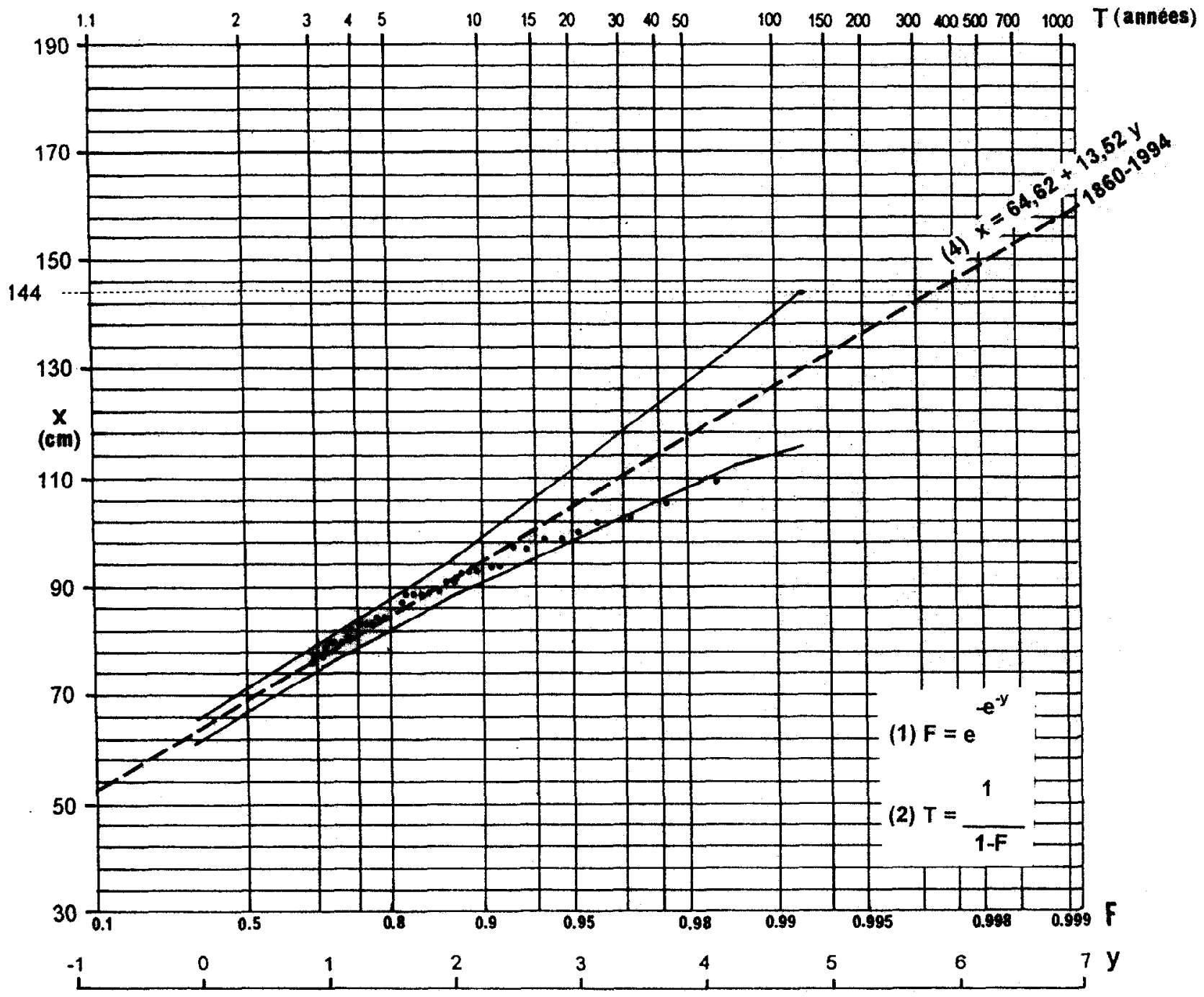

Figure 7. Fréquence des surcotes horaires maximales annuelles à Brest et limites du domaine de probabilité 2/3 (période 1860-1994). Les points correspondent aux surcotes horaires extrêmes avec périodes de retour supérieure à 3 ans.

Figure 7. Frequency of the extreme annual positive hourly sea surges at Brest and control band of $2 / 3$ probability (period 1860-1994). Dots correspond to individual extremes with return period greater than 3 years. 
de 30) au moment du pic de la surcote. Une surcote avec la même période de retour, conjuguée avec une pleine mer de vives eaux aurait pu en effet entraîner, par exemple, l'inondation des rives de la Loire entre Saint-Nazaire et Nantes [5].

Les décotes extrêmes montrent une tendance plus régulière (figure 8 ), bien qu'avec un léger déficit pour les cinq valeurs extrêmes les plus élevées, qui se situent toutes audessus de la droite d'équation (6). Bien que disposant de 125 valeurs de décotes extrêmes annuelles, la période de retour de la valeur la plus élevée $(75 \mathrm{~cm})$ ne dépasse guère 52 ans. D'un point de vue purement statistique, on peut s'attendre à ce que des décotes plus élevées (de l'ordre de 80 à $95 \mathrm{~cm}$ ) soient atteintes dans un avenir proche.

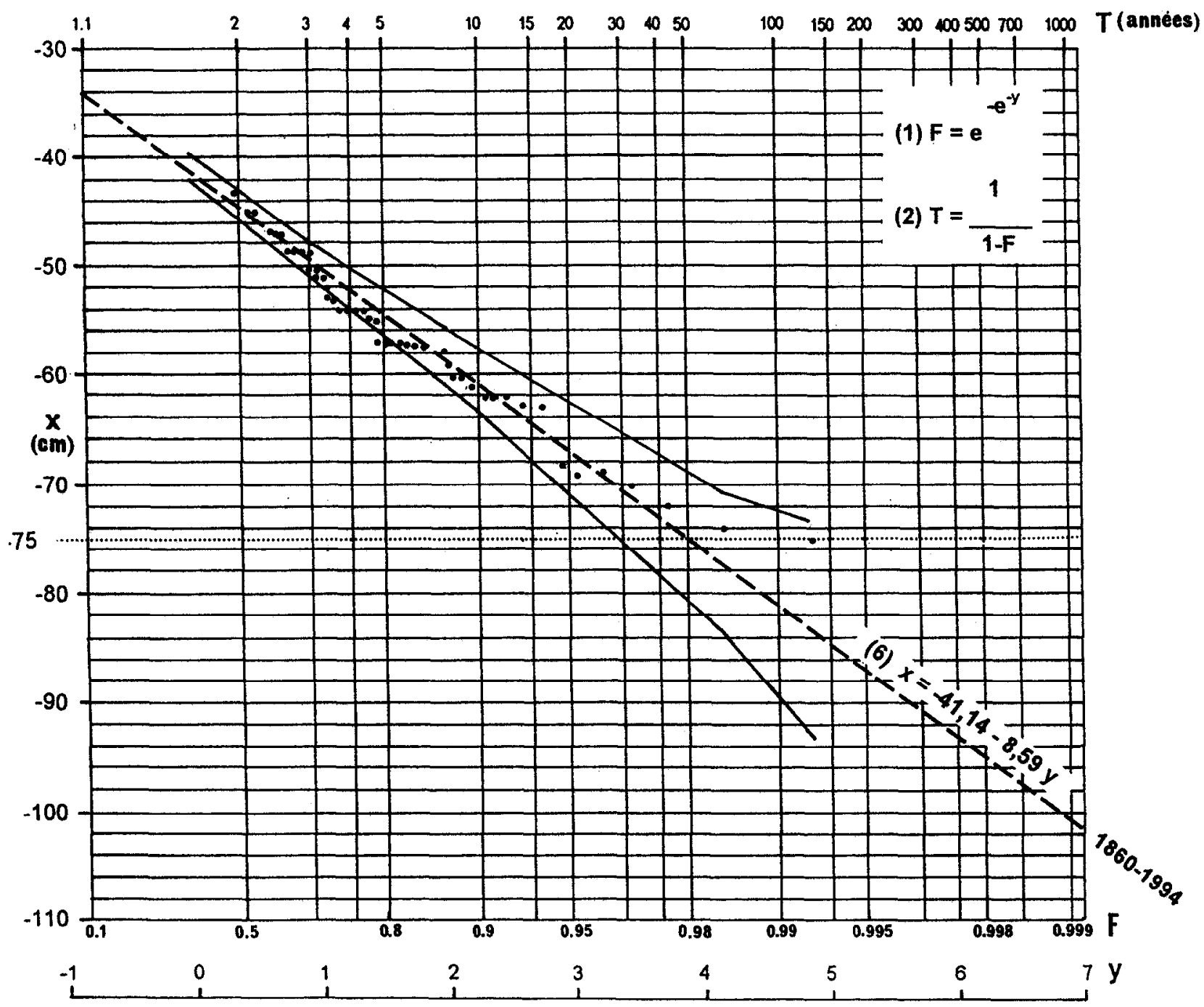

Figure 8. Fréquence des décotes horaires maximales annuelles à Brest et limites du domaine de probabilité 2/3 (période 1860-1994). Les points correspondent aux surcotes horaires extrêmes avec période de retour supérieure à 2 ans.

Figure 8. Frequency of the extreme annual negative hourly sea surges at Brest and control band of $2 / 3$ probability (period $1860-1994$ ). Dots correspond to individual extremes with return period greater than 2 years. 


\section{MÉTHODE DE SÉLECIION DES SURCOTES ET DÉCOTES SIGNIFICATIVES}

Afin de voir plus en détail comment se répartissent les surcotes et les décotes de Brest, nous avons analysé, en complétant et en affinant un travail antérieur [1], deux populations de valeurs horaires: les 1250 surcotes supérieures ou égales à $60 \mathrm{~cm}$ et les 485 décotes supérieures ou égales à $45 \mathrm{~cm}$ qui se sont produites depuis 1894 . Ce travail reposant initialement sur l'étude des corrélations entre surcotes-décotes et paramètres météorologiques [1, 2], la date initiale de 1894 a été choisie car elle correspond au début de l'archivage systématique des bulletins météorologiques quotidiens (consultables au service documentation de Météo-France).

Des deux populations considérées, il convient dans un premier temps de regrouper celles qui se trouvent suffisamment proches les unes des autres (séparées par moins de $24 \mathrm{~h}$ ) et qui n'ont pu, dans la plupart des cas, être engendrées que par le même événement météorologique. Ce premier tri permet de déterminer 206 épisodes à surcote(s), 82 épisodes à décote(s) et aucun épisode à surcote(s) et décote(s). On peut caractériser chaque épisode, entre autres, par sa hauteur maximale (surcote maximale, notée Smax ou décote maximale, notée Dmax), par sa durée totale (notée Htot) et par sa durée effective (notée $\mathrm{He}$ ), cette dernière correspondant au nombre d'heures par épisode où l'on observe effectivement une (ou des) sur$\operatorname{cote}(\mathrm{s}) \geq 60 \mathrm{~cm}$ ou bien une (ou des) décote(s) $\geq 45 \mathrm{~cm}$. Afin de ne garder que les épisodes les plus significatifs, nous n'avons retenu que les épisodes avec $\mathrm{He} \geq 6 \mathrm{~h}$, ou Smax $\geq 80 \mathrm{~cm}$ ou Dmax $\geq 65 \mathrm{~cm}$. Ce tri a abouti à la sélection de 69 épisodes à surcotes et de 19 épisodes à décotes, que nous avons considérés comme significatifs. $\mathrm{Ce}$ sont en effet les épisodes les plus durables et/ou les plus élevés qui sont potentiellement les plus dangereux pour l'environnement et les aménagements côtiers.

Cette méthode de sélection a été préférée à la méthode «du renouvellement» (souvent utilisée pour l'estimation des houles extrêmes) car, dans l'étude météorologique antérieure, elle permettait de conserver la dépendance entre pics de surcote (ou de décote) consécutifs et ainsi mieux comprendre les relations existant entre les surcotes (ou décotes) extrêmes et les conditions météorologiques associées.

De ce point de vue, les épisodes à surcotes possèdent une plus grande variabilité que les épisodes à décotes : 5 à $68 \mathrm{~h}$ de durée totale contre 7 à $52 \mathrm{~h}$, ou bien $144 \mathrm{~cm}$ de valeur maximale contre $75 \mathrm{~cm}$ pour les décotes. Plus les épisodes sont longs et plus grande est la probabilité que des surcotes (ou des décotes) s'ajoutent à une pleine mer (ou à une basse mer) et produisent des hauts (ou bas) niveaux d'eau historiques. Parmi les épisodes significatifs à surcotes, on peut distinguer, en fonction de leur durée, deux populations principales relativement homogènes: 49 épisodes courts, entre 5 et 24 h (notés de type A) et 20 épisodes longs, entre 25 et $68 \mathrm{~h}$ (notés de type B). Les épisodes de type A sont en moyenne trois fois plus courts que les épisodes de type B ; ils sont aussi plus continus et

Tableau I. Typologie et principaux paramètres analytiques des épisodes significatifs à surcotes $\geq 60 \mathrm{~cm}$ ou à décotes $\geq 45 \mathrm{~cm}$ (série marégraphique de Brest 1894-1995).

Table I. Typology and defined analytic parameters of selected positive or negative sea surges events (tide-gauge data for Brest 1894-1995).

\begin{tabular}{|c|c|c|c|c|}
\hline & & \multicolumn{2}{|c|}{69 épisodes à surcotes } & \multirow[t]{2}{*}{19 épisodes à décotes } \\
\hline & & Type A (43) & Type B (18) & \\
\hline Durée totale (Htot) & Minimum & 5 & 25 & 14 \\
\hline \multirow[t]{3}{*}{ (h) } & Maximum & 24 & 68 & 52 \\
\hline & Moyenne & 12 & 37 & 29 \\
\hline & Écart type & 5 & 12 & 11 \\
\hline Durée effective (He) & Minimum & 5 & 5 & 6 \\
\hline \multirow[t]{3}{*}{ (h) } & Maximum & 21 & 58 & 26 \\
\hline & Moyenne & 10 & 21 & 11 \\
\hline & Écart type & 5 & 13 & 6 \\
\hline \multirow{4}{*}{$\begin{array}{l}\text { Hauteur maximale } \\
(\text { Smax ou Dmax) } \\
(\mathrm{cm})\end{array}$} & Minimum & 64 & 68 & 49 \\
\hline & Maximum & 144 & 99 & 75 \\
\hline & Moyenne & 83 & 81 & 55 \\
\hline & Écart type & 14 & 9 & 4 \\
\hline
\end{tabular}


Tableau II. Analyse comparative des épisodes à surcotes $\geq 60 \mathrm{~cm}$ et à décotes $\geq 45 \mathrm{~cm}$ observés à Brest pour les périodes 1894-1944 et 1953-1995.

Table II. Comparative analysis of positive and negative sea-surge events observed at Brest between 1894-1944 and 1953-1995.

\begin{tabular}{lcc}
\hline Période & $\mathbf{1 8 9 4 - 1 9 4 4}$ & $\mathbf{1 9 5 3 - 1 9 9 5}$ \\
Années utiles & $\mathbf{4 8}$ & $\mathbf{4 1}$ \\
\hline Épisodes à surcotes : & & \\
$\quad$ Effectifs du type A & 26 & 22 \\
Effectifs du type B & 6 & 14 \\
Effectif moyen annuel (A+B) & 0,7 & 0,9 \\
$\begin{array}{l}\text { Durée moyenne des épisodes } \\
\text { (h) }\end{array}$ & 17 & 22 \\
Hauteur moyenne des épisodes & 82 & 83 \\
(cm) & & \\
Épisodes à décotes : & 10 & \\
Effectif total & 0,2 & 0,2 \\
Effectif moyen annuel & 33 & 26 \\
$\begin{array}{l}\text { Durée moyenne des épisodes } \\
\text { (h) }\end{array}$ & & \\
Hauteur moyenne des épisodes & 55 & 56 \\
(cm) & & \\
\hline
\end{tabular}

légèrement plus intenses. Concernant les décotes, en revanche, aucun type particulier n'a pu être différencié (tableau I).

\section{RÉPARTITION INTRA- ET INTERANNUELLE DES ÉCHANTILLONS DÉFINIS}

L'évolution de la fréquence annuelle des épisodes significatifs à surcotes $\geq 60 \mathrm{~cm}$ ct à décotcs $\geq 45 \mathrm{~cm}$ depuis 1894 est synthétisée dans le tableau II et sur la figure 9. Cette évolution ne se présente pas de façon progressive et linéaire, mais selon des alternances entre des phases d'amplification et de régression. Ces phases, pour les surcotes, ont des durées assez constantes, comprises entre 11 et 16 ans (tableau III); la fréquence moyenne annuelle apparaît particulièrement forte entre 1910 et 1919 et entre 1954 et 1966 (à chaque fois : 16 épisodes à surcotes $\geq 60 \mathrm{~cm}$ ). Quant aux épisodes à décotes, bien que leur nombre soit insuffisant pour établir des statistiques fiables, leur fréquence semble subir peu d'évolution entre les deux périodes comparatives 1894-1944 et 1953-1995 (tableau II). Les années à forte fréquence de surcotes sont le plus souvent associées à des années présentant peu ou pas de décotes $(1912,1914,1966,1989)$. Il en cst de même pour les décotes $(1905,1917,1934)$. Mais à l'échelle décennale, aucune association n'apparaît régulièrement, les phases d'amplification ou de régression des épisodes à décotes étant parfois simultanées de celles des épisodes à surcotes, mais aussi en déphasage (tableau III et figure 9).

Tableau II. Phases d'évolution des surcotes horaires $\geq 60 \mathrm{~cm}$, des décotes horaires $\geq 45 \mathrm{~cm}$ à Brest et des épisodes associés (1894-1995).

Table III. Evolution phases of $\geq 60 \mathrm{~cm}$ positive and $\geq 45 \mathrm{~cm}$ negative hourly sea surges and joint events at Brest (1894-1995).

\begin{tabular}{|c|c|c|c|c|c|c|c|c|c|}
\hline Surcotes : Période & $\leq 1894-1909$ & 1909-1924 & 1924-1934 & $1934 \geq 1944$ & $\leq 1953-1965$ & 1965-1977 & 1977-1988 & $1988 \longrightarrow 1995$ & 1894-1995 \\
\hline $\begin{array}{l}\text { Durée de la phase } \\
\text { (années) }\end{array}$ & $\geq 16$ & 16 & 11 & $\geq 11$ & $\geq 13$ & 13 & 12 & $\geq 8$ & \\
\hline $\begin{array}{l}\text { Nb. moyen/an } \\
S \geq 60 \mathrm{~cm}\end{array}$ & 5,0 & 20,5 & 6,0 & 16,0 & 15,5 & 14,4 & 12,0 & 15,1 & 13,5 \\
\hline Smax moyenne $(\mathrm{cm})$ & 65,6 & 75,3 & 68,3 & 69,4 & 76.7 & 74,8 & 75,5 & 81,1 & 72,9 \\
\hline Nb. d'épisodes & 5 & 16 & 4 & 8 & 12 & 9 & 10 & 7 & 69 \\
\hline Type de phase & Basse & Haute & Basse & (Moyenne) & (Haute) & Haute & Moyenne & Haute & \\
\hline Décotes : Période & $\leq 1894-1899$ & 1899-1915 & 1915-1932 & 1932-1942 & $1942-\geq 1944$ & $\leq 1953-1973$ & $1973-1988$ & $1988 \rightarrow 1995$ & 1894-1995 \\
\hline $\begin{array}{l}\text { Durée de la phase } \\
\text { (années) }\end{array}$ & $\geq 6$ & 17 & 18 & 11 & $\geq 3$ & $\geq 21$ & 16 & $\geq 8$ & \\
\hline $\begin{array}{l}\text { Nb. moyen/an } \\
\mathrm{D} \geq 45 \mathrm{~cm}\end{array}$ & 0,0 & 3,6 & 3,8 & 3,8 & 3,7 & 3,3 & 5,4 & 0,1 & 3,5 \\
\hline Dmax moyenne $(\mathrm{cm})$ & 41,0 & 46,0 & 45,6 & 45,6 & 48,0 & 44,5 & 51,3 & 37,0 & 45,0 \\
\hline $\begin{array}{l}\text { Nb. d'épisodes } \\
\text { Type de phase }\end{array}$ & $\begin{array}{l}0 \\
\text { Rasce }\end{array}$ & $4:$ & 4 & 1 & 1 & 5 & $\begin{array}{l}4 \\
\text { Haute }\end{array}$ & $\begin{array}{l}0 \\
\text { Basse }\end{array}$ & 19 \\
\hline
\end{tabular}

Phase basse quand $\mathrm{Nb}$. moyen/an $\mathrm{S} \geq 60$ (D $\geq 45$ ) et $S \max$ (Dmax) moyenne de la phase sont inférieurs aux moyennes de la période 1894-1995. Phase haute quand $\mathrm{Nb}$. moyen/an $\mathrm{S} \geq 60(\mathrm{D} \geq 45)$ et $\mathrm{Smax}$ (Dmax) moyenne de la phase sont supérieurs aux moyennes de la période 18941995.

Phase moyenne quand les deux indices se comportent différemment par rapport à la période 1894-1995. 


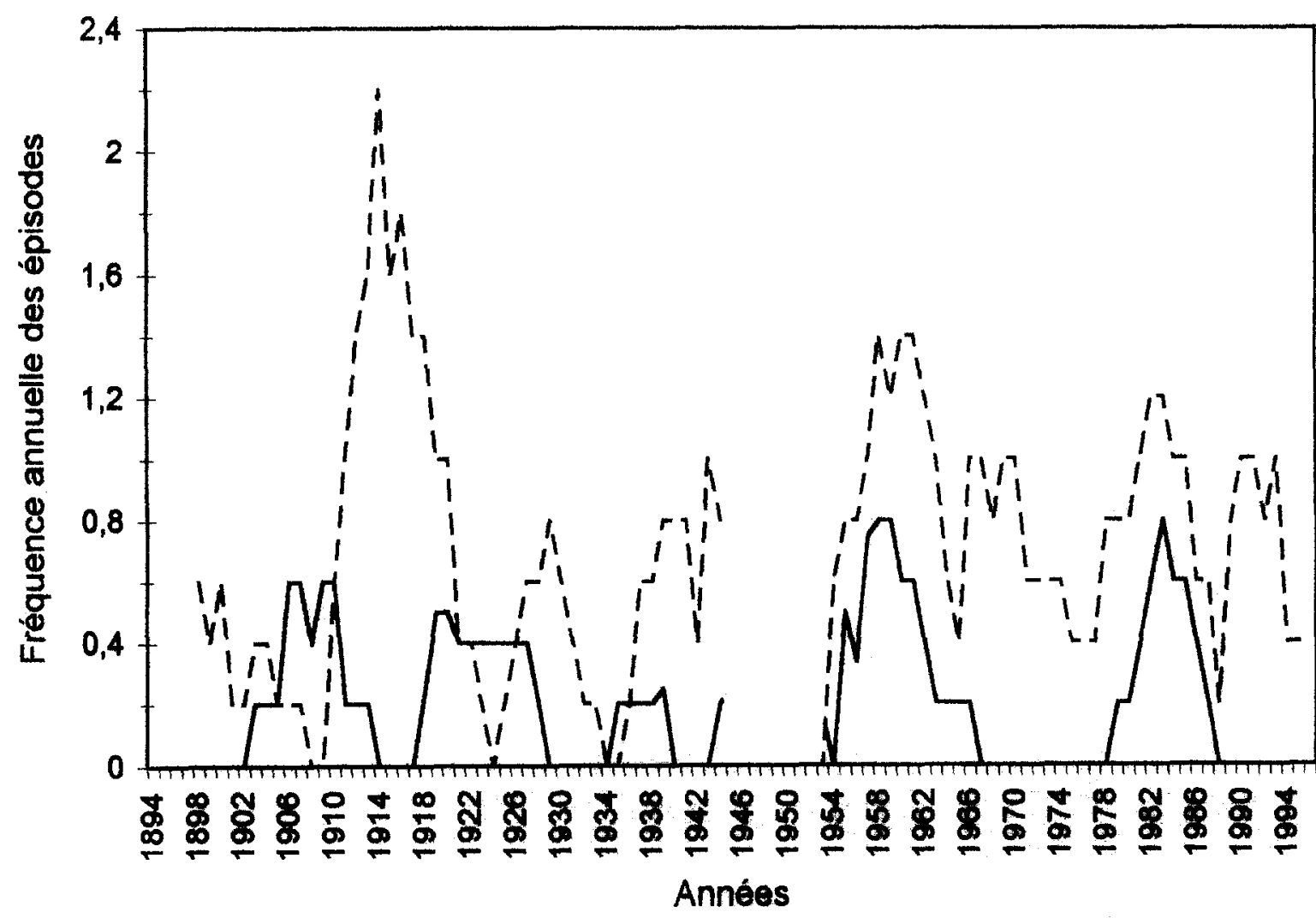

- - - Epis. à surcotes (moyenne sur 5 ans) ——Epis. à décotes (moyenne sur 5 ans)

Figure 9. Fréquences annuelles des épisodes à surcotes $\geq 60 \mathrm{~cm}$ et à décotes $\geq 45 \mathrm{~cm}$ à Brest (1894-1995).

Figure 9. Annual distributions of positive $(\geq 60 \mathrm{~cm})$ and negative $(\geq 45 \mathrm{~cm})$ sea surge events at Brest (1894-1995).

La répartition et la fréquence intra-annuelle des surcotes $\geq 60 \mathrm{~cm}$ et des épisodes à surcotes (figure 10) montrent une opposition très nette entre la période hivernale (décembre à février), où il se produit plus de $80 \%$ de ces phénomènes, et la saison estivale (juin à août), avec seulement trois surcotes $\geq 60 \mathrm{~cm}$ depuis 1894 . Décembre ( $36 \%$ des cas) et février ( $22 \%$ ) apparaissent comme les mois les plus favorables de l'année. La période hivernale est également la plus favorable au développement des décotes $\geq 45 \mathrm{~cm}$ et des épisodes associés (figure 10), avec, pour ces derniers, des mois de janvier (33\%) et février $(30 \%)$ très largement prédominants; mais l'opposition été-hiver y est moins sensible. D'évidence, les situations météorologiques de saison froide réunissent plus fréquemment les conditions nécessaires à la génération des surcotes et des décotes.

\section{CONCLUSION}

L'étude des principales caractéristiques des surcotes et des décotes horaires enregistrées à Brest depuis 1860 ne permet pas de distinguer une tendance nette de variation à long terme de ces phénomènes, quel que soit le type de valeurs analysées (valeurs moyennes, extrêmes ou subextrêmes). En revanche, leur évolution peut se décomposer en plusieurs pas de temps. À court terme d'abord, on observe une variabilité interannuelle très forte. À moyen terme, ensuite, cette évolution, bien que non linéaire, s'organise en une succession de phases d'amplification et de régression, de durées assez constantes pour les surcotes (cycles de 11 à 16 ans) et plus variables pour les décotes, mais sans association particulière entre ces niveaux extrêmes ambivalents. Enfin, à long terme, il n'existe pas 

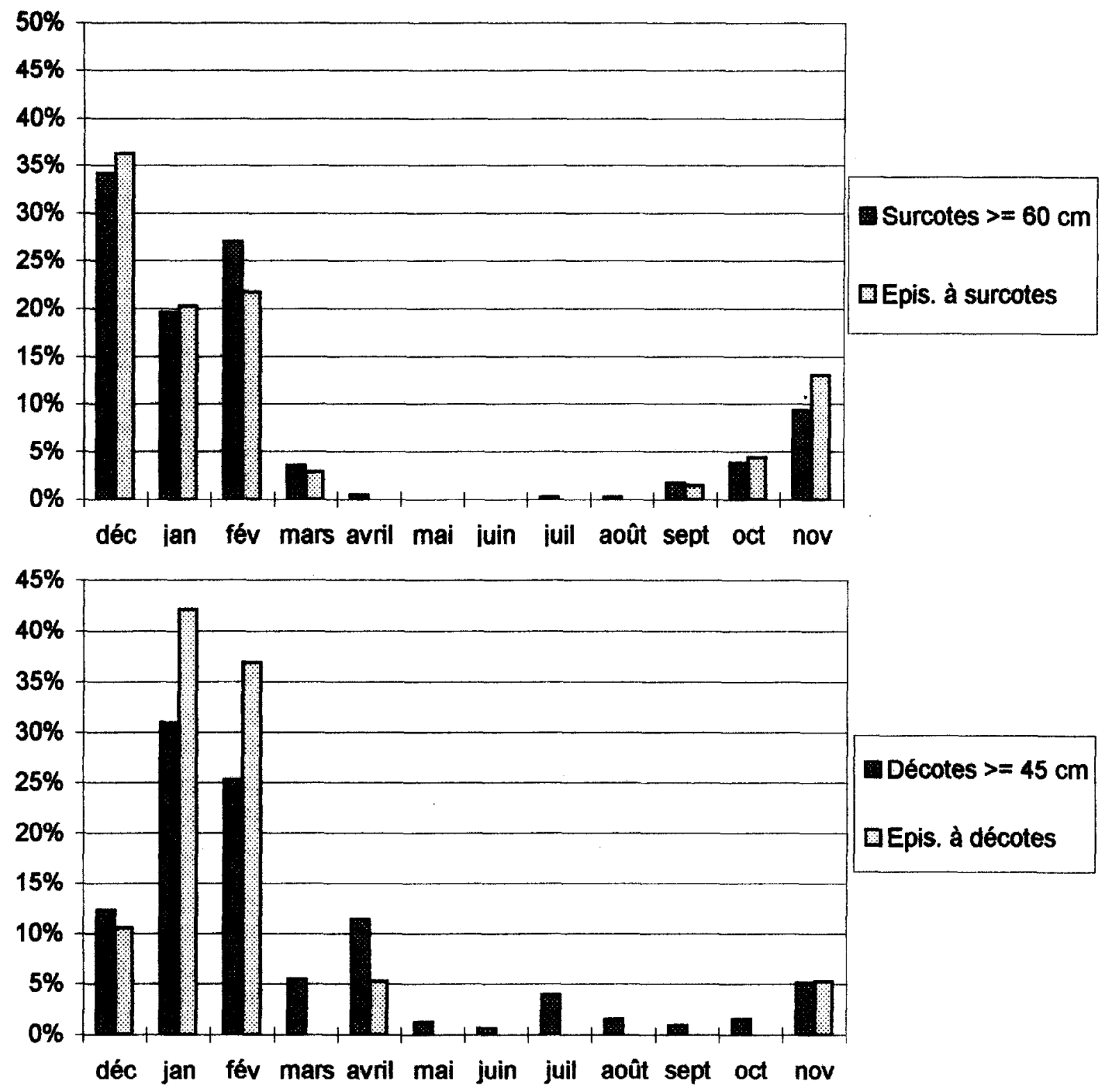

Figure 10. Répartition mensuelle des surcotes $\geq 60 \mathrm{~cm}$, des décotes $\geq 45 \mathrm{~cm}$ et des épisodes associés à Brest (1894-1995).

Figure 10. Monthly distribution of positive sea surges $(\geq 60 \mathrm{~cm})$, negative sea-surges $(\geq 45 \mathrm{~cm})$ and joint events at Brest (1894-1995).

de tendance claire ; néanmoins, il faut retenir que la fréquence des surcotes extrêmes a augmenté pour la période récente (1953-1994), alors que les décotes (extrêmes ou subextrêmes) ont suivi une tendance opposée, ce qui a pour effet de renforcer la prédominance déjà forte des surcotes sur les décotes pour les valeurs les plus élevées.

Les échelles temporelles de variations de ces manifestations océaniques correspondent à celles à l'origine de ces phénomènes. En effet, bien qu'elles soient atypiques et éphémères en regard de l'évolution globale du niveau des mers, elles restent en étroite relation avec la circulation atmosphérique générale et donc avec ses échelles de variations temporelles [1]. Par conséquent, l'étude de ces niveaux extrêmes devient d'autant plus intéressante qu'ils peuvent servir d'indicateurs de l'évolution globale du climat, au même titre que le niveau moyen des 
mers (mais pour des échelles spatio-temporelles plus réduites).

\section{Remerciements}

Cette étude est une contribution au projet de la Commission européenne «Storms » (DG12-VOMA : contrat
EN V4-CT97-0488). Les auteurs remercient l'ingénieur B. Simon du service hydrographique et océanographique de la marine, pour les facilités apportées lors du dépouillement des données marégraphiques et le Professeur M. Tabeaud de l'université de Paris-1, pour son aide lors des recherches menées et dans la préparation de ce travail.

\section{RÉFÉRENCES}

[1] Bouligand R., Surcotes et décotes à Brest depuis 1894, étude phénoménologique et évolution, DEA, université d'Orléans (1997) $171 \mathrm{p}$.

[2] Bouligand R., Tabeaud M., Cent ans de surcotes à Brest, Met. Mar. 180 (1998) 12-16.

[3] Darchen J., Ondes et marées de tempêtes, exemples d'interaction océan-atmosphère, Met. Mar. 91 (1976) 25-31.

[4] Douglas B.C., Global sea level acceleration, J. Geophys. Res. 97 (C8) (1992) 12699-12706.

[5] Ghadri H., La marée de l'ingénieur civil, prévision des surcotes, thèse École centrale Nantes, (1994) 168 p.

[6] Gumbel E.J., Statistical theory of extreme values and some practical applications, a series of lectures, U.S. Dep. Commerce, Nat. Bur. Standards, Applied Mathematics Ser. (1954) 33 , viii $+51 \mathrm{p}$.

[7] Pirazzoli P.A., Valori estremi di marea nella laguna di Venezia, Tecnica Italiana 35 (9) (1970) 433-446.
[8] Pirazzoli P.A., Maree estreme a Venezia (periodo 1872-1981), Acqua \& Aria 10 (1982) 1023-1039.

[9] Pugh D.T., Vassie J.M., Extreme sea levels from tide and surge probability, Proc. 16th Coastal Engineering Conf. Hamburg. Am. Soc. Civil Eng. New York, 1 (1979) 911-930.

[10] Regnauld H., Dubreuil V., L'élévation du niveau marin dans l'Ouest français: signification climatique et conséquences morphologiques, Ann. Géogr. 600 (1998) 117-138.

[11] Simon B., Statistique des niveaux marins extrêmes le long des côtes de France, Shom Brest, Rapp. ét. 001/94 DPNM (ministère de l'Équipement) (1994) $78 \mathrm{p}$.

[12] Town J.A., Vassie J.M., Extreme sea levels, The joint probabilities method revisited and revised, Proceedings of Institute of Civil Engors, part 2, (87) (1989) 429-442.

[13] Woodworth P.L., A search for accelerations in records of European mean sea level, Int. J. Climatol. 10 (1990) 129-143. 